\title{
Relações sinérgicas e efeitos sobre a produção setorial no sistema inter-regional Paraná-Restante do Brasil
}

\author{
Rossana Lott Rodrigues ${ }^{1}$ \\ Antonio Carlos Moretto ${ }^{2}$ \\ Umberto Antonio Sesso Filho ${ }^{3}$ \\ Ricardo Kureski ${ }^{4}$
}

Resumo: O presente artigo tem como objetivo estimar e analisar o nível das interações sinérgicas e o transbordamento do efeito multiplicador da produção setorial entre o Paraná e o chamado "Restante do Brasil" (Brasil exceto Paraná), utilizando sistemas inter-regionais de insumoproduto estimados para os anos de 1995 e 2000. Os principais resultados mostraram que: a) a dependência da produção do Paraná em relação ao fluxo de comércio com o Restante do Brasil se elevou de $25 \%$ em 1995 para $33 \%$ em 2000; b) somente $1,36 \%$ da produção do Restante do Brasil dependeu do fluxo de insumos (bens e serviços) entre este e o Paraná em 1995, reduzindo esta dependência para 0,56\% em 2000; c) a média do transbordamento do efeito multiplicador da produção dos setores da economia paranaense no sentido Restante do Brasil aumentou de $16 \%$ em 1995 para $20 \%$ em 2000, enquanto no sentido Restante do Brasil-Paraná, se manteve em 1\%. Diante desses resultados, o poder público estadual, por meio de políticas que visem

\footnotetext{
${ }^{1}$ Doutora em Economia Aplicada pela Esalq/USP e professora do Departamento de Economia da Universidade Estadual de Londrina. E-mail: rlott@uel.br ${ }^{2}$ Doutor em Economia Aplicada pela Esalq/USP e professor do Departamento de Economia da Universidade Estadual de Londrina. E-mail: acmoretto@uel.br

${ }^{3}$ Doutor em Economia Aplicada pela Esalq/USP e professor do Departamento de Economia da Universidade Estadual de Londrina. E-mail: umasesso@uel.br

${ }^{0}$ Doutor em Economia e Política Florestal pela Universidade Federal do Paraná (UFPR), pesquisador do Instituto Paranaense de Desenvolvimento Econômico e Social (Ipardes), investigador do Laboratório de Contabilidade Social da UFPR e professor da Pontifícia Universidade Católica do Paraná (PUC/PR). E-mail: kureski@pr.gov.br
} 
melhorar a infra-estrutura estadual e de políticas de estímulos setoriais, poderá colaborar, criando o aparato logístico e designando incentivos fiscais e/ou creditícios específicos que auxiliariam a iniciativa privada na implementação de suas atividades, de modo a tornar mais eficiente e vantajoso o comércio inter-regiões.

Palavras-chave: insumo-produto; interações sinérgicas; transbordamento da produção; economia regional.

Abstract: The present article has as objective to calculate and to analyze the level of the synergic interactions and the overflow of the multiplying effect of the sectorial production between Parana and the Rest of Brazil (Brazil except Parana), using interregional systems of input-output estimated for the years of 1995 and 2000. The main results showed that: a) the dependence of the production of Parana in relation to the flow of trade with the Rest of Brazil raised from $25 \%$ in 1995 to $33 \%$ in 2000 ; b) only $1.36 \%$ of the production of the Rest of Brazil depended on the flow of input (goods and services) between this and Parana in 1995 , reducing this dependence to $0,56 \%$ in 2000 ; c) the overflowing average of the multiplier effect of the production of the sectors of the economy of Parana in direction of the Rest of Brazil raised from 16\% in 1995 to $20 \%$ in 2000, while in direction of the Rest of Brazil-Parana, it kept on $1 \%$.

Key-words: input-output; synergic interactions; overflow of the production; regional economy.

JEL Classification: C67, D57, R15

\section{Introdução}

O processo de globalização em curso tende a reforçar as relações entre as economias, tanto no sentido da troca das informações quanto no que se refere ao comércio de bens e serviços e ao fluxo de capitais. No Brasil, a economia começa os anos 90 sob a égide dos programas de liberalização comercial que, desde meados de 
1980, vêm, progressivamente, sendo implantados. A competição mais acirrada, resultante do processo de abertura econômica, exige a modernização e a reestruturação dos setores produtivos nacionais e regionais. Essa exigência encontra, no Brasil e no Paraná, diferenças no que se referem à infra-estrutura, ao parque produtivo, ao capital humano, à distribuição de renda, etc., o que requer grande esforço local para sua superação.

Com o objetivo de situar a economia paranaense relativamente à do Restante do Brasil, a Tabela 1 apresenta três importantes indicadores, os quais revelam que o Paraná é um estado equilibrado no que se refere ao número de habitantes e produtos, apresentando renda per capita maior que a do Restante do Brasil e a nacional em 2000.

Tabela 1 - Estatísticas do Paraná, Restante do Brasil e Brasil, 2000.

\begin{tabular}{cccccc}
\hline Regiões & $\begin{array}{c}\text { PIB } \\
\text { (milhões R\$) }\end{array}$ & $\begin{array}{r}\text { PIB } \\
(\%)\end{array}$ & População & $\begin{array}{c}\text { População } \\
(\%)\end{array}$ & $\begin{array}{c}\text { PIB per capita } \\
(\mathrm{R} \$)\end{array}$ \\
\hline Paraná & 65.969 & 5,99 & 9.585 .731 & 5,63 & 6.882 \\
$\begin{array}{c}\text { Restante } \\
\text { do Brasil }\end{array}$ & 1.035 .286 & 94,01 & 160.544 .809 & 94,37 & 6.449 \\
Brasil & $\mathbf{1 . 1 0 1 . 2 5 5}$ & $\mathbf{1 0 0 , 0 0}$ & $\mathbf{1 7 0 . 1 3 0 . 5 4 0}$ & $\mathbf{1 0 0 , 0 0}$ & $\mathbf{6 . 4 7 3}$ \\
\hline
\end{tabular}

Fonte: Ipardes (2004).

Quanto à estrutura econômica, o Paraná vem se transformando rapidamente ao longo das últimas décadas. Embora as bases para essas transformações tenham sido lançadas na década de 1970, a consolidação de alguns setores ocorreu nos anos 90, com a abertura comercial da economia brasileira. Um exemplo é a indústria automobilística, que recebeu incentivos fiscais, financeiros e de infra-estrutura do governo do estado para instalação de empresas na região de Curitiba, com previsão de surgimento de novos empregos e aumento da produção. Hoje, a estrutura do parque industrial automotivo paranaense possui características próximas à de outros centros nacionais, embora apresente, como principais entraves, a pouca tradição de fornecimento, a retração do mercado consumidor e o grande poder concorrencial do setor (Pinto e Meza, 2003). 
Outros setores da economia paranaense foram acionados a partir de 1995, com a política de incentivos do governo estadual, como os de metalurgia, madeira e mobiliário, celulose, papel e gráfica, têxtil, vestuário, calçados, couros e peles, alimentar, serviços públicos, construção civil (industrial e residencial), transporte e comunicação, gerando mudanças importantes no processo produtivo e nas relações com o mercado.

O ajuste da base produtiva do Paraná promoveu a concentração patrimonial e produtiva em alguns setores e regiões do estado, resultando, ainda, em impactos negativos sobre os postos de trabalho, conforme Oliveira (2003).

Segundo Alves (1997), dos R4 8,6 bilhões de investimentos em andamento em 1996, o setor metalmecânico absorveu 56\%, cabendo a maior parte $(24,3 \%$ ou cerca de $\mathrm{R} \$ 2,1$ bilhões) às montadoras de automóveis. No caso específico do setor automobilístico, os efeitos esperados sobre a economia estadual, no que se referem à geração de empregos, não se confirmaram, gerando dúvidas sobre os benefícios advindos da política implementada (Motim et al., 2004).

Em relação à concentração espacial, a forte predominância dos investimentos na região de Curitiba pode ser vista na Tabela 2.

No entanto, as transformações em curso no Paraná trouxeram a modernização da estrutura produtiva do estado, com novos produtos, processos e tecnologias, além de dinamizar as relações com outros mercados, tornando mais complexo, dinâmico e diversificado o perfil industrial paranaense.

Diante dessa nova realidade, a integração entre as regiões torna-se fator sine qua non para o melhor desempenho do Paraná no atual cenário mundial, uma vez que a auto-suficiência não é possível, nem desejável, do ponto de vista econômico. A maior interação entre diferentes regiões promove, concomitantemente, maiores efeitos sinérgicos e dependência econômica entre estruturas produtivas diferentes. $\mathrm{O}$ aumento da produção setorial em determinada região do Brasil tem impacto sobre a produção de diversas indústrias fora da localidade de origem, o que se chama efeito transbordamento do multiplicador de produção. Portanto, a mensuração das interações sinérgicas e do efeito transbordamento do multiplicador de produção torna-se importante 
fonte de informações para a análise do desenvolvimento regional e elaboração de políticas públicas, identificando as indústrias que apresentam maior dependência de insumos provenientes de outras regiões.

Tabela 2 - Distribuição regional dos investimentos no Paraná, 1996.

\begin{tabular}{lrrr} 
& & & Em \% \\
\hline Gêneros & Região de Curitiba & Demais Regiões & Total \\
\hline Agroindústria & 19,52 & 80,48 & 100 \\
Minerais não-metálicos & 100 & 0 & 100 \\
Metalurgia & 88,89 & 11,11 & 100 \\
Mecânica & 95,38 & 4,62 & 100 \\
Material elétrico e comunicação & 96,75 & 3,25 & 100 \\
Material de transporte & 100 & 0 & 100 \\
Madeira/mobiliário & 9,52 & 90,48 & 100 \\
Química & 86,25 & 13,75 & 100 \\
Papel e papelão & 0 & 100 & 100 \\
Produtos de matérias plásticas & 48,28 & 51,72 & 100 \\
Perfumaria & 100 & 0 & 100 \\
Bebidas & 24,32 & 75,68 & 100 \\
Gráfico & 100 & 0 & 100 \\
Total & $\mathbf{7 0 , 2 3}$ & $\mathbf{2 9 , 7 7}$ & $\mathbf{1 0 0}$ \\
\hline
\end{tabular}

Fonte: Alves (1997).

Alguns trabalhos foram desenvolvidas para o Brasil com o objetivo de estudar a sinergia entre regiões, como os de Guilhoto et al. (1998), Guilhoto et al. (1999), Guilhoto et al. (2001), e/ou o transbordamento do multiplicador de produção (Sesso et al., 2003). Para o Paraná, estudos sobre sinergia foram realizados por Moretto (2000) e Simões et al. (2003). No entanto, o efeito transbordamento do multiplicador de produção ainda não foi avaliado entre o Paraná e o Restante do Brasil.

Assim, o objetivo desta pesquisa é mensurar o nível das interações sinérgicas resultantes do fluxo de bens e serviços e o transbordamento do efeito multiplicador da produção setorial entre o Paraná e o Restante do Brasil, comparando os anos de 1995 e 2000, período de grande transformação na estrutura produtiva estadual. Especificamente, pretende-se: 
a) estimar as interações sinérgicas entre o Paraná e o Restante do Brasil (RBR);

b) estimar o transbordamento do multiplicador tipo I da produção setorial do Paraná e do Restante do Brasil e

c) realizar comparações dos indicadores econômicos (transbordamento e sinergia) entre as regiões.

Este artigo está estruturado em 3 seções, além da introdução. Inicialmente, é exposta a metodologia. Posteriormente, são analisados os resultados e, por fim, apresentadas as considerações finais.

\section{Metodologia}

\subsection{Fonte dos dados}

Este estudo tem como fonte de dados as matrizes insumo-produto estimadas para o Paraná em 1995 por Rodrigues (2000) e Moretto (2000) e em 2000 por Nuñes et al. (2003), além da matriz insumo-produto nacional de 1995, construída pelo IBGE (1997), e de 2000, estimada por Guilhoto et al. (2002).

A partir das matrizes, foram estimados os sistemas inter-regional de insumo-produto Paraná-Restante do Brasil para 1995 e $2000^{5}$.

\subsection{Métodos de Análise}

\subsubsection{Interações sinérgicas entre regiões}

A metodologia relativa às interações sinérgicas foi desenvolvida por Sonis et al. (1997) e possibilita examinar, por meio das interdependências internas e externas dadas pelas ligações, a estrutura das relações comerciais entre duas regiões. Está baseada num sistema de insumoprodução partilhado e utiliza técnicas que produzem multiplicadores à esquerda e à direita da inversa de Leontief, dentro de um preestabelecido par de combinações hierárquicas dos subsistemas de ligações econômicas.

${ }^{5}$ O sistema inter-regional Paraná-Restante do Brasil já foi usado em outros trabalhos, como Simões et al. (2003), Sesso et al. (2004) e Rodrigues et al. (2005). 
Considerando-se um sistema de insumo-produção representado pelo bloco de matrizes, $A$, de insumos diretos:

$$
A=\left[\begin{array}{ll}
A_{11} & A_{12} \\
A_{21} & A_{22}
\end{array}\right]
$$

em que $A_{11}$ e $A_{22}$ representam matrizes quadradas de insumos diretos dentro da primeira e segunda regiões, respectivamente, e $A_{12}$ e $A_{21}$ são matrizes retangulares dos insumos diretos adquiridos pela segunda região e vice-versa, é possível interpretar a matriz A como um sistema de duas regiões em que a segunda região representa o restante da economia menos a primeira região.

A construção dos blocos de pares de combinações hierárquicas dos subsistemas de ligações intra e inter-regionais, num sistema de insumoprodução, é dada pelas matrizes $A_{11}, A_{12}, A_{21}$ e $A_{22}$, as quais correspondem a quatro blocos básicos de matrizes:

$$
A_{l l}=\left[\begin{array}{cc}
A_{l l} & 0 \\
0 & 0
\end{array}\right] ; \quad A_{l 2}=\left[\begin{array}{cc}
0 & A_{l 2} \\
0 & 0
\end{array}\right] ; \quad A_{2 l}=\left[\begin{array}{cc}
0 & 0 \\
A_{2 l} & 0
\end{array}\right] ; \quad A_{22}=\left[\begin{array}{cc}
0 & 0 \\
0 & A_{22}
\end{array}\right]
$$

A decomposição do bloco de matrizes (1) pode ser feita por meio da soma de dois blocos de matrizes, sendo cada um deles a soma dos blocos de matrizes de (2). Dessa forma, pode ser apresentado um conjunto de multiplicadores regionais internos, derivados das matrizes inversas, as quais são blocos construídos das interações sinérgicas entre os subsistemas econômicos. O uso das diferentes interações sinérgicas possibilita analisar e mensurar como ocorrem as transações entre regiões. Assim, é possível verificar o quanto as relações de produção em uma dada região afetam a produção de outra região.

O Quadro 1 e a Figura 1 mostram, respectivamente, as possíveis interações sinérgicas e as combinações das partes da matriz $A_{1}$. Cada entrada no Quadro 1 consiste de dois níveis: o primeiro descreve a estrutura e mostra a correspondente forma da matriz $A_{1}$, enquanto o segundo mostra as decomposições aditivas da matriz bloco de Leontief. A visão do sistema de hierarquias de ligações 
fornecerá novas interpretações das propriedades das estruturas que são reveladas.

Além disso, Sonis et al. (1997), Guilhoto et al. (1998) e Guilhoto et al. (1999) sugerem a seguinte tipologia de categorias que podem ser empregadas:

1. tipo de ligação para trás (VI, IX): poder de dispersão;

2. tipo de ligação para frente $(\mathrm{V}, \mathrm{X})$ : sensibilidade de dispersão;

3. tipo de ligações intra e inter-regionais (VII, VIII): dispersão interna e externa;

4. estilo de interações de região isolada versus o restante da economia (I, XIV, IV, XI) e

5. estilo de subsistema triangular versus as interações inter-regionais (II, XIII, III, XII).

Assim, os sistemas de insumo-produção partilhados podem diferenciarse entre os vários tipos de dispersão (como 1, 2 e 3) e entre os vários modelos de interações inter-regionais (como 4 e 5). Essencialmente, as 5 categorias e os 14 tipos de pares de combinações hierárquicas de ligações econômicas propiciam a oportunidade de escolher de acordo com as qualidades especiais das atividades de cada região e com o tipo de problema que se apresenta, evidenciando que as opções existem para as bases de uma tipologia dos tipos de economia baseados na estrutura hierárquica (Quadro 1).

Quadro 1 - Ordenação das interações sinérgicas entre os subsistemas econômicos.

(Continua)

Nível 1

Descrição

Forma da matriz $A_{1}$

Nível $2 L=L_{1}+\left(M_{L}-I\right) L_{1}=L_{1}+L_{l}\left(M_{R}-I\right)$

I. Hierarquia da região isolada versus o restante da economia

$$
L=\left[\begin{array}{cc}
B_{l} & 0 \\
0 & I
\end{array}\right]+\left[\begin{array}{cc}
B_{I} A_{l 2} & 0 \\
0 & I
\end{array}\right] D_{2}\left[\begin{array}{cc}
I & I \\
I & I-S_{2}
\end{array}\right]\left[\begin{array}{cc}
A_{2 l} B_{l} & 0 \\
0 & I
\end{array}\right] \quad A_{l}=\left[\begin{array}{cc}
A_{l l} & 0 \\
0 & 0
\end{array}\right]
$$


II. A ordem da hierarquia substituída das ligações inter-regionais da segunda região versus o subsistema triangular inferior

$$
L=\left[\begin{array}{cc}
I & A_{I 2} \\
0 & I
\end{array}\right]+\left[\begin{array}{cc}
D_{I} & 0 \\
0 & D_{2}
\end{array}\right]\left[\begin{array}{cc}
I-S_{l} & A_{I 2} B_{2}-S_{l} A_{l 2} \\
A_{2 I} B_{l} & I-S_{2}
\end{array}\right] \quad A_{I}=\left[\begin{array}{cc}
0 & A_{12} \\
0 & 0
\end{array}\right]
$$

III. A ordem da hierarquia substituída das ligações inter-regionais da primeira região versus o subsistema triangular superior

$$
L=\left[\begin{array}{cc}
I & 0 \\
A_{2 I} & I
\end{array}\right]+\left[\begin{array}{cc}
D_{l} & 0 \\
0 & D_{2}
\end{array}\right]\left[\begin{array}{cc}
I-S_{l} & A_{l 2} B_{2} \\
A_{2 I} B_{I}-S_{2} A_{2 I} & I-S_{2}
\end{array}\right] \quad A_{l}=\left[\begin{array}{cc}
0 & 0 \\
A_{2 l} & 0
\end{array}\right]
$$

IV. A ordem da hierarquia substituída das ligações para trás e para frente da primeira região versus o restante da economia

$$
L=\left[\begin{array}{cc}
I & 0 \\
0 & B_{2}
\end{array}\right]+\left[\begin{array}{cc}
I & 0 \\
0 & B_{2} A_{2 l}
\end{array}\right] D_{l}\left[\begin{array}{cc}
I-S_{l} & I \\
I & I
\end{array}\right]\left[\begin{array}{cc}
I & 0 \\
0 & A_{12} B_{2}
\end{array}\right] \quad A_{1}=\left[\begin{array}{cc}
0 & 0 \\
0 & A_{22}
\end{array}\right]
$$

V. Hierarquia das ligações para frente da primeira e da segunda regiões

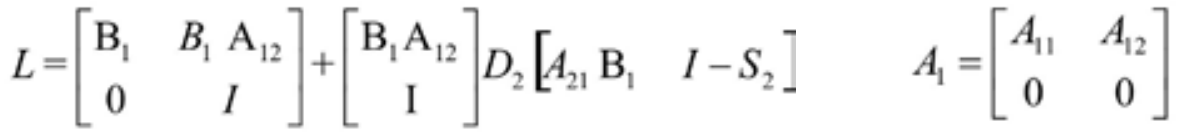

VI. Hierarquias das ligações para trás da primeira e segunda regiões

$$
L=\left[\begin{array}{cc}
\mathrm{B}_{1} & 0 \\
\mathrm{~A}_{21} \mathrm{~B}_{l} & I
\end{array}\right]+\left[\begin{array}{c}
\mathrm{B}_{1} \mathrm{~A}_{12} \\
\mathrm{I}-\mathrm{S}_{2}
\end{array}\right] D_{2}\left[\begin{array}{lll}
A_{21} \mathrm{~B}_{1} & I_{-}^{-}
\end{array} \quad A_{1}=\left[\begin{array}{cc}
A_{11} & 0 \\
A_{2 I} & 0
\end{array}\right]\right.
$$

VII. Hierarquia das relações intra versus inter-regionais

$$
A_{1}=\left[\begin{array}{cc}
A_{11} & 0 \\
0 & A_{22}
\end{array}\right]
$$

$L=\left[\begin{array}{cc}\mathrm{B}_{1} & 0 \\ 0 & \mathrm{~B}_{2}\end{array}\right]+\left[\begin{array}{ccc}D_{1} \mathrm{~A}_{12} \mathrm{~B}_{2} & 0 \\ 0 & D_{2} \mathrm{~A}_{21} \mathrm{~B}_{1}\end{array}\right]\left[\begin{array}{cc}\mathrm{A}_{21} & \mathrm{I}-\mathrm{A}_{22} \\ \mathrm{I}-\mathrm{A}_{11} & \mathrm{~A}_{12}\end{array}\right]\left[\begin{array}{cc}B_{1} & 0 \\ 0 & \mathrm{~B}_{2}\end{array}\right]$

VIII. Hierarquia das relações inter versus intra-regionais

$$
\mathrm{A}_{1}=\left[\begin{array}{cc}
0 & A_{12} \\
A_{21} & 0
\end{array}\right]
$$


$L=\left[\begin{array}{cc}\mathrm{D}_{11}^{*} & \mathrm{D}_{11}^{*} \mathrm{~A}_{12} \\ \mathrm{D}_{22}^{*} \mathrm{~A}_{21} & \mathrm{D}_{22}^{*}\end{array}\right]+\left[\begin{array}{cc}\mathrm{I} & \mathrm{B}_{1} \mathrm{~A}_{12} \\ \mathrm{~B}_{2} \mathrm{~A}_{21} & \mathrm{I}\end{array}\right]\left[\begin{array}{cc}\mathrm{D}_{1} \mathrm{~A}_{11} \mathrm{D}_{11}^{*} & 0 \\ 0 & \mathrm{D}_{2} \mathrm{~A}_{22} \mathrm{D}_{22}^{*}\end{array}\right]\left[\begin{array}{cc}\mathrm{I} & \mathrm{A}_{12} \\ \mathrm{~A}_{21} & \mathrm{I}\end{array}\right]$

IX. Ordem de hierarquia substituída de ligações para trás

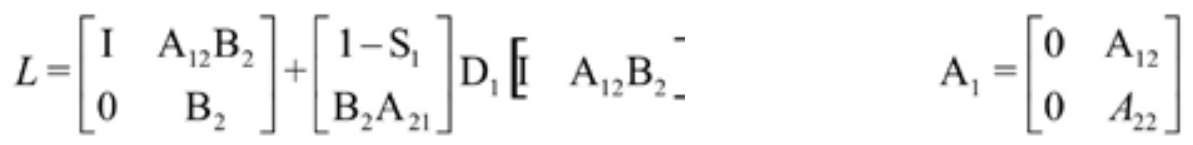

$\mathbf{X}$. Ordem de hierarquia substituída de ligações para frente

$$
L=\left[\begin{array}{cc}
\mathrm{I} & 0 \\
\mathrm{~B}_{2} \mathrm{~A}_{21} & \mathrm{~B}_{2}
\end{array}\right]+\left[\begin{array}{c}
1 \\
\mathrm{~B}_{2} \mathrm{~A}_{21}
\end{array}\right] \mathrm{D}_{1}\left[\begin{array}{ll}
\mathrm{I}-\mathrm{S}_{1} & \mathrm{~A}_{12} \mathrm{~B}_{2}
\end{array}\right] \quad \mathrm{A}_{1}=\left[\begin{array}{cc}
0 & 0 \\
A_{21} & A_{22}
\end{array}\right]
$$

XI. Hierarquia das ligações para trás e para frente da primeira região versus o restante da economia

$$
L=\left[\begin{array}{cc}
\mathrm{D}_{1}^{*} & \mathrm{D}_{1}^{*} \mathrm{~A}_{12} \\
\mathrm{~A}_{21} \mathrm{D}_{1}^{*} & \mathrm{D}_{22}
\end{array}\right]+\left[\begin{array}{c}
\mathrm{B}_{1} \mathrm{~A}_{12} \\
\mathrm{I}
\end{array}\right] \mathrm{D}_{2} \mathrm{D}_{22} \mathrm{~A}_{22}\left[\begin{array}{ll}
\mathrm{A}_{21} \mathrm{~B}_{1} & \mathrm{I}
\end{array}\right] \mathrm{A}_{1}=\left[\begin{array}{cc}
\mathrm{A}_{11} & \mathrm{~A}_{12} \\
\mathrm{~A}_{21} & 0
\end{array}\right]
$$

XII. Hierarquia do subsistema triangular superior versus as ligações inter-regionais da primeira região

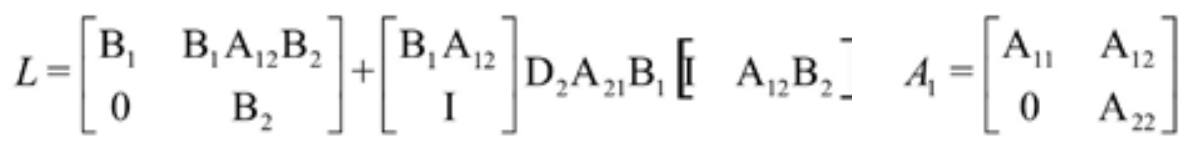

XIII. Hierarquia do subsistema triangular inferior versus ligações inter-regionais da segunda região

$$
L=\left[\begin{array}{cc}
\mathrm{B}_{1} & 0 \\
\mathrm{~B}_{2} \mathrm{~A}_{21} \mathrm{~B}_{1} & \mathrm{~B}_{2}
\end{array}\right]+\left[\begin{array}{c}
1 \\
\mathrm{~B}_{2} \mathrm{~A}_{21}
\end{array}\right] \mathrm{D}_{1} \mathrm{~A}_{12} \mathrm{~B}_{2}\left[\begin{array}{ll}
\mathrm{A}_{21} \mathrm{~B}_{1} & \mathrm{I}_{-}^{-}
\end{array} \mathrm{A}_{1}=\left[\begin{array}{cc}
\mathrm{A}_{11} & 0 \\
\mathrm{~A}_{21} & \mathrm{~A}_{22}
\end{array}\right]\right.
$$

XIV. Hierarquia do restante da economia versus a segunda região isolada

$$
L=\left[\begin{array}{cc}
\mathrm{D}_{11} & \mathrm{~A}_{12} \mathrm{D}_{2}^{*} \\
\mathrm{D}_{2}^{*} \mathrm{~A}_{21} & \mathrm{D}_{2}^{*}
\end{array}\right]+\left[\begin{array}{c}
1 \\
\mathrm{~B}_{2} \mathrm{~A}_{21}
\end{array}\right] \mathrm{D}_{11} \mathrm{~A}_{11} \mathrm{D}_{1}\left[\begin{array}{lll}
\mathrm{I} & \mathrm{A}_{12} \mathrm{~B} & 2_{-}^{-}
\end{array} \quad \mathrm{A}_{1}=\left[\begin{array}{cc}
0 & \mathrm{~A}_{12} \\
\mathrm{~A}_{21} & \mathrm{~A}_{22}
\end{array}\right]\right.
$$

Fonte: Sonis et al. (1997). 
Figura 1 - Representação esquemática das formas possíveis da matriz $A_{1}$ - o caso de duas regiões.
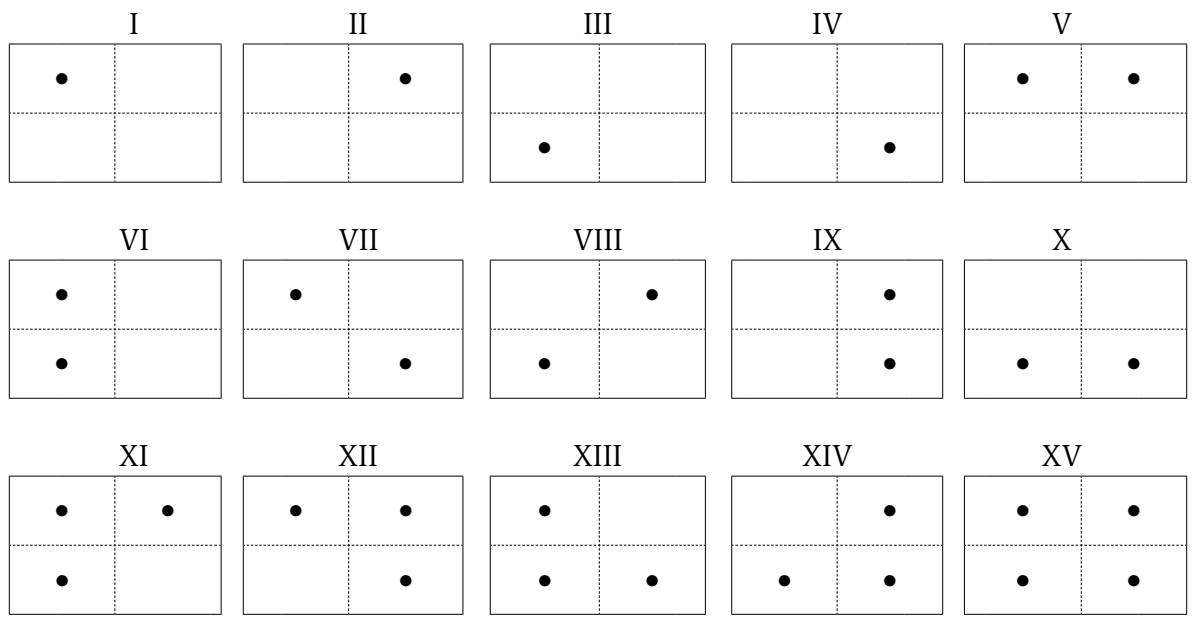

Fonte: Moretto (2000).

\subsubsection{Efeito transbordamento do multiplicador de produção}

Para estimar o efeito transbordamento do multiplicador da produção é necessário, primeiramente, calcular o multiplicador, o qual permite analisar o impacto da variação na demanda final de determinado setor sobre a variável econômica de interesse (Miller e Blair, 1985).

No presente estudo, a variável usada será a produção. Assim, dado que $L=(I-A)^{-1}$ é a matriz inversa de Leontief, o multiplicador setorial de produção do setor $j$ será:

$$
M P_{j}=\sum_{i=1}^{n} l_{i j}, \quad j=1, \ldots, n
$$

em que $\mathrm{l}_{\mathrm{ij}}$ representa os elementos da matriz inversa de Leontief e $\mathrm{MP}_{\mathrm{j}}$ é o multiplicador de produção do tipo I.

O multiplicador representa o valor total de produção de toda a economia, que é acionado para atender à variação de uma unidade na demanda final do setor $j$. Como este estudo é desenvolvido para 32 setores, $\mathrm{i}=\mathrm{j}=64$. 
Assim, o somatório dos elementos da matriz inversa referente à própria região constitui o efeito multiplicador interno, enquanto o somatório dos elementos da coluna j referente ao fluxo inter-regional de bens e serviços é o valor do transbordamento (efeito multiplicador fora da região de origem do setor). Como pode ser observado na equação (1), os elementos $1_{\mathrm{ij}}$ da matriz L $\mathrm{L}^{\mathrm{ll}}$, somados em colunas, são o efeito multiplicador dentro da região L, enquanto os somatórios das colunas da matriz $\mathrm{L}^{\mathrm{ML}}$ são transbordamentos dos setores da região L para a região $\mathrm{M}$.

$\mathrm{O}$ transbordamento do efeito multiplicador de dado setor de uma região em relação à outra pode ser apresentado tanto em termos absolutos quanto em valores percentuais. O efeito transbordamento mostra como o aumento da produção setorial em dada região impacta a produção dos setores de outra região.

\section{Resultados e discussão}

\subsection{Interações sinérgicas e transbordamento do efeito multiplicador da produção entre o Paraná e o Restante do Brasil}

Os resultados das interações sinérgicas para o Paraná e o Restante do Brasil para os anos de 1995 e 2000 estão resumidos na Figura 2. Ao analisá-los, nota-se que a produção do Paraná se tornou mais dependente do fluxo de comércio entre este e o Restante do Brasil de 1995 para 2000.

Em 1995, a interação entre o Paraná e o restante do país determinou que, cerca de, $25 \%$ da produção paranaense dependessem do fluxo de bens e serviços em direção ao Restante do Brasil e 7,5\% do nível da produção nacional. Já em 2000, esses valores se elevam para cerca de $33 \%$ e $9 \%$, respectivamente.

Paralelamente, o Restante do Brasil, que já apresentava elevada importância nas próprias relações intersetoriais para sua produção em 1995 (cerca de 98\%), reforça essa posição em 2000, quando 99\% da produção é determinada pelas ligações entre os setores de sua economia. Além disso, somente 1,36\% da produção do Restante do Brasil dependeu do fluxo de insumos (bens e serviços) entre este e o Paraná em 1995, reduzindo essa dependência para 0,56\% em 2000 (Figura 2). 
Figura 2 - Contribuição (\%) de cada bloco de matriz para a participação total de $\left(y_{1}-f\right)$ em y, Paraná (PR) e Restante do Brasil (RBR), 1995 e 2000.

Sistema Paraná-Restante do

Brasil em 1995

\begin{tabular}{|c|c|c|}
\hline \multicolumn{3}{|c|}{ Paraná } \\
\hline & PR & RBR \\
\hline PR & 67,08 & 25,31 \\
\hline RBR & 0,12 & 7,49 \\
\hline
\end{tabular}

Sistema Paraná-Restante do

Brasil em 2000

Paraná

\begin{tabular}{c|c|c|}
\multicolumn{1}{c}{ Paraná } \\
\multicolumn{1}{c}{ PR } & RBR \\
\cline { 2 - 3 } PR & 58,23 & 32,82 \\
\cline { 2 - 3 } RBR & 0,04 & 8,91 \\
\cline { 2 - 3 } & &
\end{tabular}

Restante do Brasil

\begin{tabular}{c|c|c|c}
\multicolumn{1}{c}{} & \multicolumn{1}{c}{ PR } & \multicolumn{2}{c}{ RBR } \\
\cline { 2 - 3 } PR & 0,34 & 0,09 & PR \\
RBR & 1,36 & 98,21 & RBR \\
\cline { 2 - 3 } & & &
\end{tabular}

\begin{tabular}{|c|c|c|}
\hline & \multicolumn{2}{|l|}{$\mathrm{Re}$} \\
\hline & PR & RBR \\
\hline PR & 0,16 & 0,08 \\
\hline RBR & 0,56 & 99,20 \\
\hline
\end{tabular}

Fonte: Cálculo dos autores.

A comparação dos resultados obtidos para os anos em tela indica que o Paraná aumentou a interação com os outros estados do País. Pode-se afirmar que houve relativo aumento do fluxo de insumos no sentido Restante do Brasil-Paraná, resultando em maior dependência da estrutura produtiva (consumo intermediário) deste estado em relação ao restante do País, ao mesmo tempo em que houve redução das vendas de insumos das empresas paranaenses para o restante do Brasil. Em 2000, as relações intersetoriais no Paraná eram responsáveis por $58,23 \%$ da produção, contra 67,08\% em 1995 (Figura 2).

A maior interação sinérgica indica, ao mesmo tempo, a abertura da economia estadual e sua relativa dependência em relação aos fluxos de bens e serviços utilizados no consumo intermediário. Portanto, pode-se prever que o Paraná deve apresentar maior transbordamento do efeito multiplicador da produção setorial que o Restante do Brasil. Assim, o aumento global da produção estadual implicará na necessidade de incremento do fluxo de comércio entre este estado e o restante do País e o conseqüente aumento da produção em outras 
localidades, gerando o efeito transbordamento do multiplicador de produção.

O efeito do transbordamento do multiplicador de produção é mostrado, em valores e em percentuais, nas Tabelas 3 e 4 e ilustrado nas Figuras 3, 4, 5 e 6.

A análise dos resultados aponta que, se os setores do Paraná aumentassem sua produção, em média, $16 \%$ e $20 \%$ do efeito multiplicador, em 1995 e 2000, respectivamente, teria impacto fora do estado. Por outro lado, o transbordamento no sentido Restante do Brasil-Paraná não ultrapassaria $1 \%$ nos dois anos. Diante disso, pode-se afirmar que o Paraná apresentou crescimento de sua dependência em relação ao Restante do País e, ao mesmo tempo, elevação do efeito transbordamento (Tabela 3).

A análise mais detalhada mostra que o transbordamento do multiplicador de produção Paraná-Restante do Brasil para 1995 foi maior para os setores Siderurgia e Metalurgia (4) e Indústria da Borracha (10), sendo que o aumento da produção dessas atividades no Paraná geraria um efeito multiplicador de produção, fora da região de origem, que variaria entre $34 \%$ e $40 \%$, em média. Por outro lado, se ocorresse aumento de produção dos setores alimentares (16 a 21), Madeira e mobiliário (8), Celulose, papel e gráfica (9) e Serviços industriais de utilidade pública (24) no Restante do Brasil, haveria um transbordamento do efeito multiplicador da produção dentro do Paraná de $3 \%$ (Tabela 3 e Figuras 3 e 4).

Para 2000, o transbordamento do multiplicador de produção ParanáRestante do Brasil mostrou que os setores Siderurgia e Metalurgia (4), Fabricação de elétrico/eletrônicos (6), Equipamentos de transporte e peças (7), Indústria têxtil (14) e Vestuário, calçados, couros e peles (15) apresentaram os maiores valores, indicando que o aumento da produção dessas atividades no Paraná geraria um efeito multiplicador de produção fora da região de origem que variaria entre $31 \%$ e $44 \%$ (Tabela 4 e Figuras 5 e 6).

Em contrapartida, houve redução do transbordamento do efeito multiplicador de produção do Restante do Brasil para o Paraná em 2000. Assim, se ocorresse aumento de produção dos setores alimentares (18, 19, 21 e 22), Madeira e mobiliário (8) e Farmacêutica, veterinária e perfumaria (12) no Restante do Brasil, haveria transbordamento do efeito multiplicador da produção dentro do Paraná que variaria de $2 \%$ a $5 \%$ (Tabela 4 e Figuras 5 e 6). 
Tabela 3 - Transbordamento do multiplicador de produção do sistema interregional Paraná-Restante do Brasil, 1995.

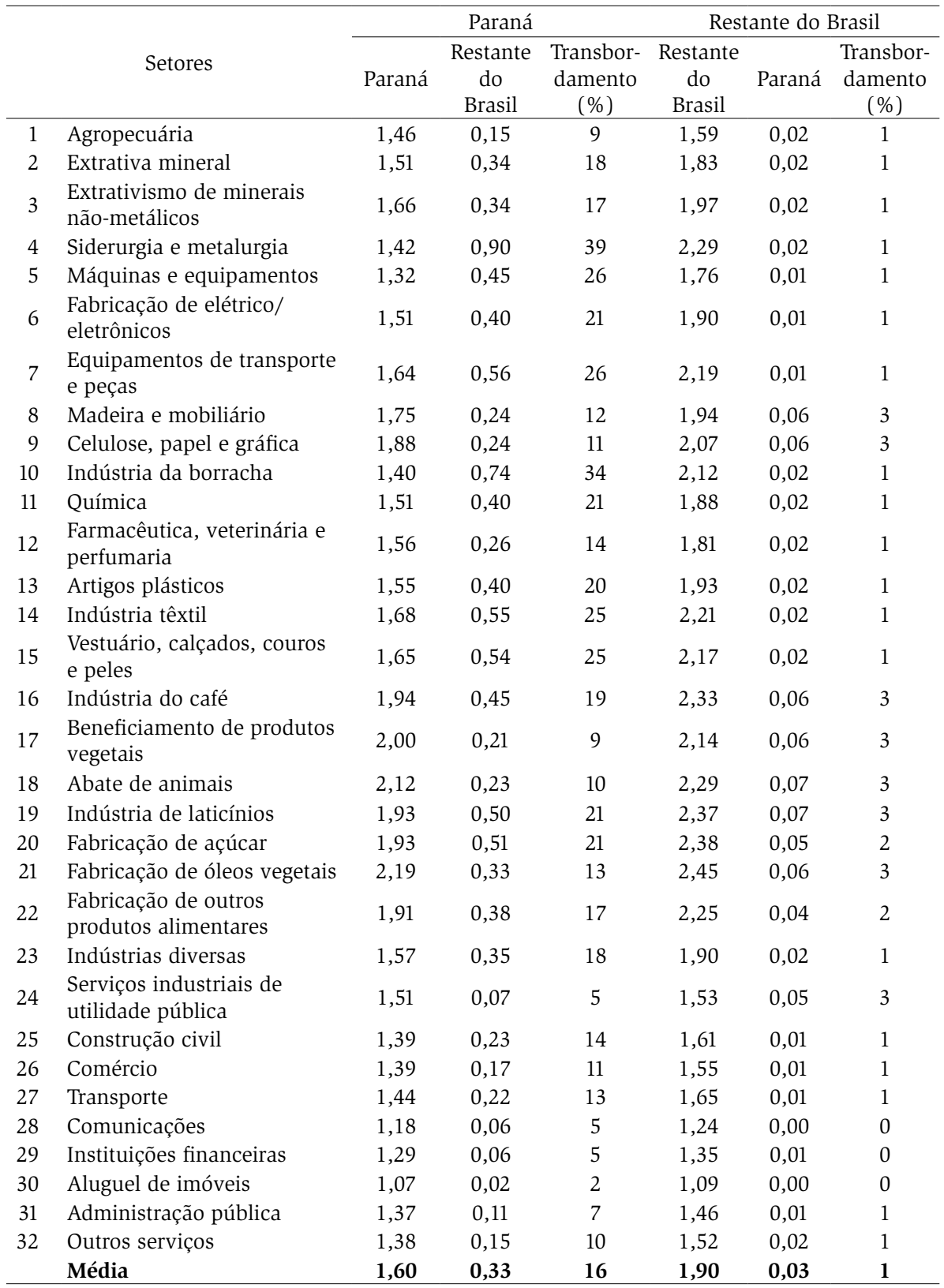

Fonte: Cálculo dos autores. 
Tabela 4 - Transbordamento do multiplicador de produção do sistema interregional Paraná-Restante do Brasil, 2000.

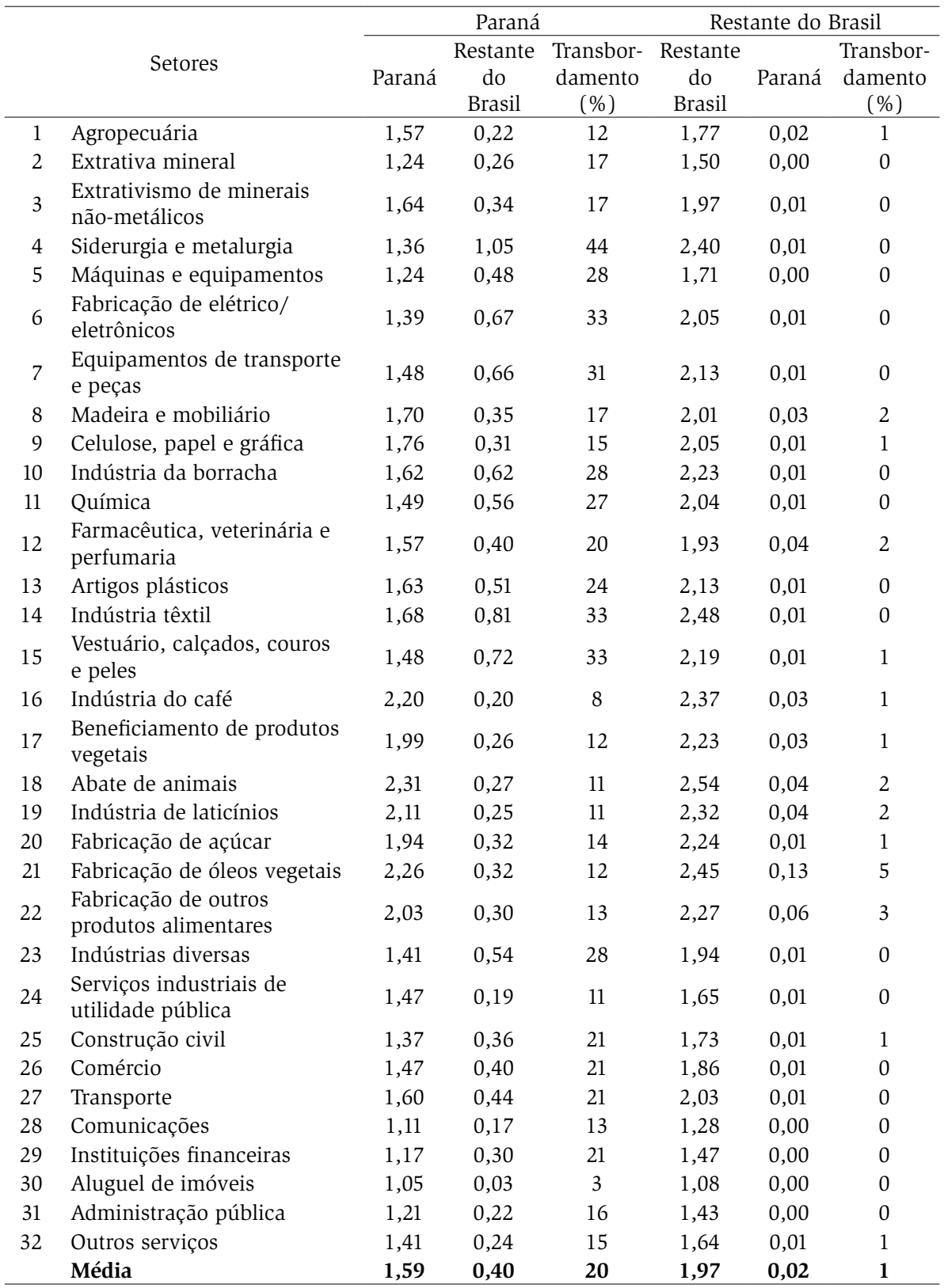

Fonte: Cálculo dos autores. 


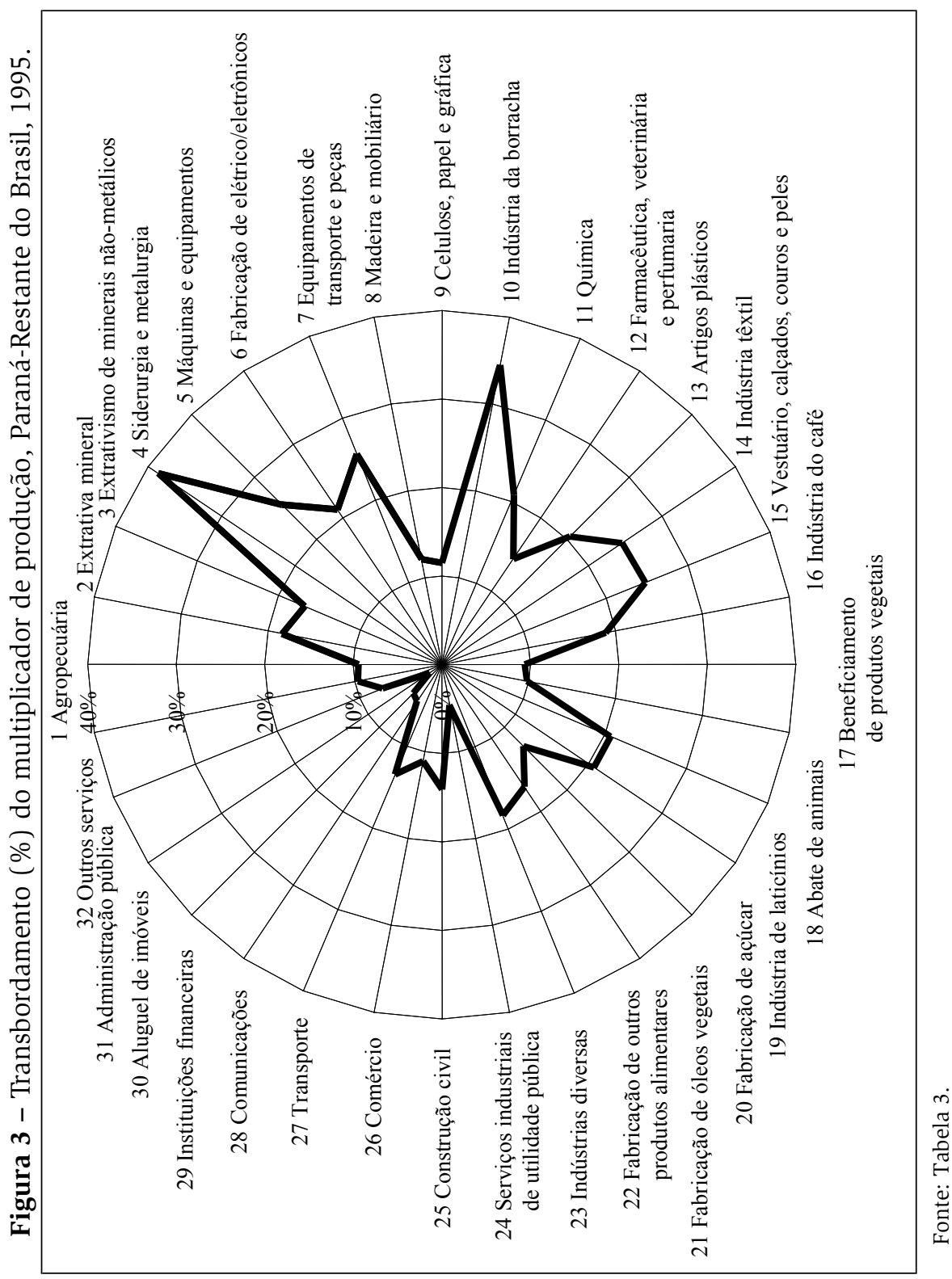




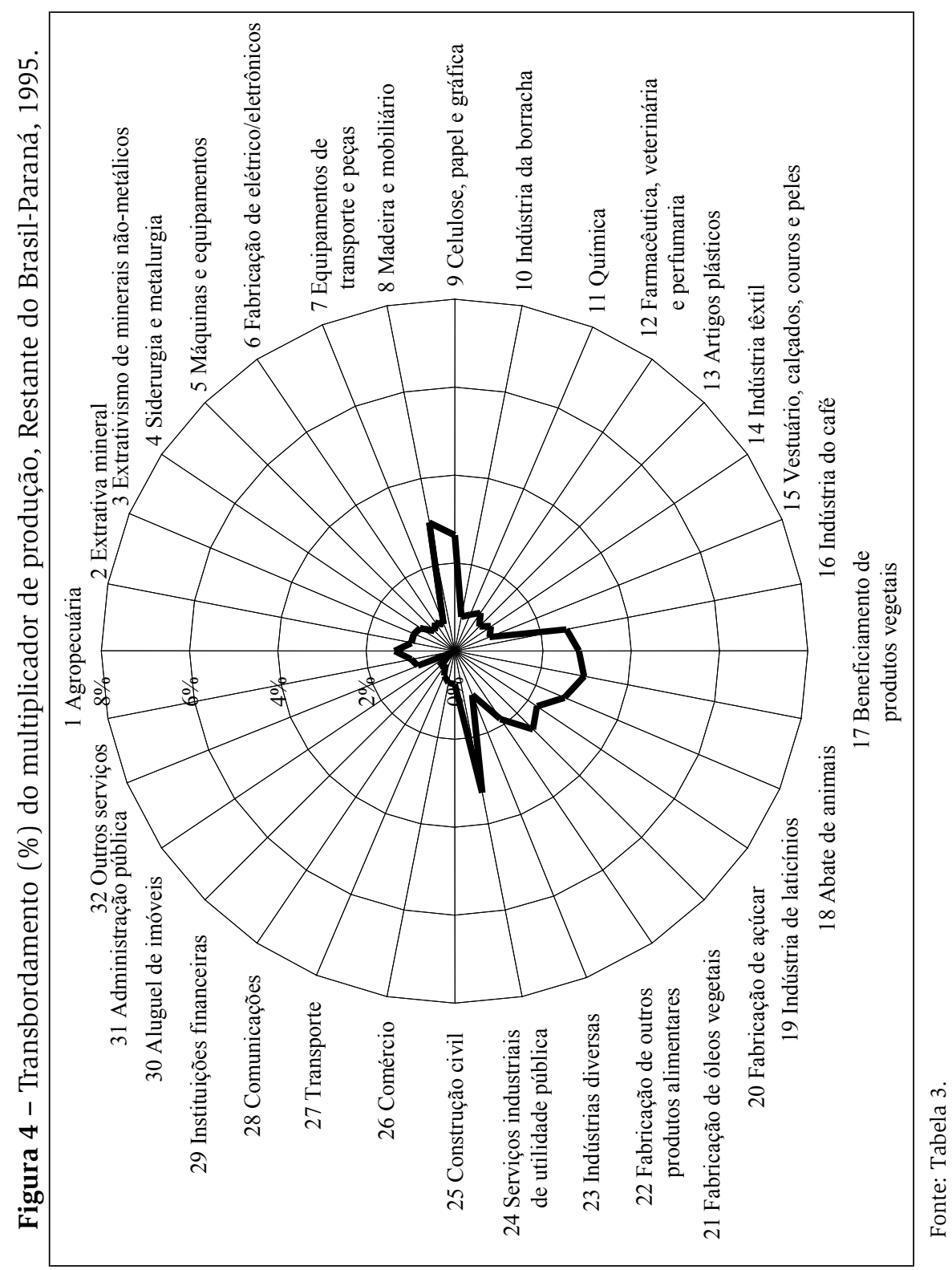




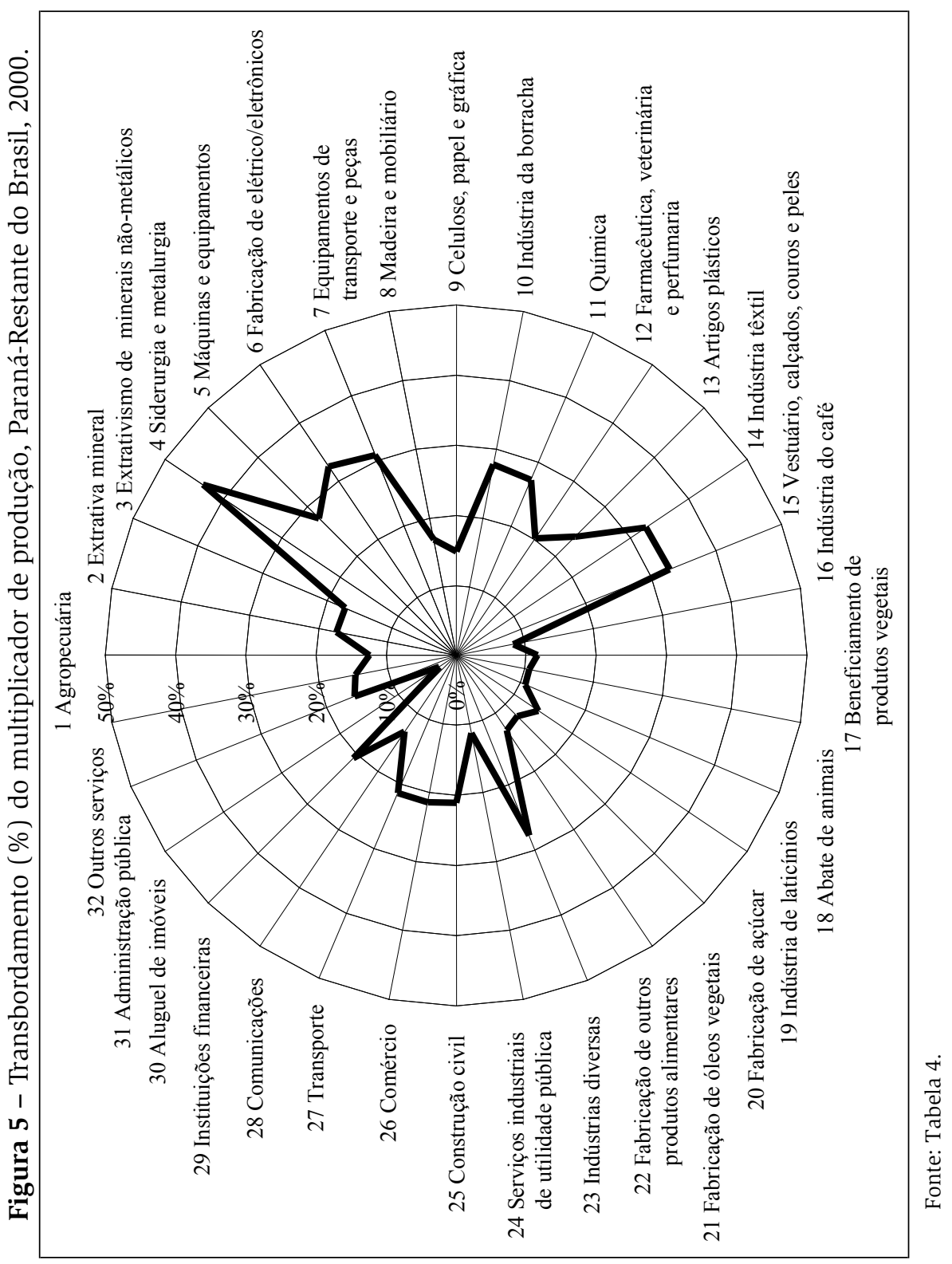




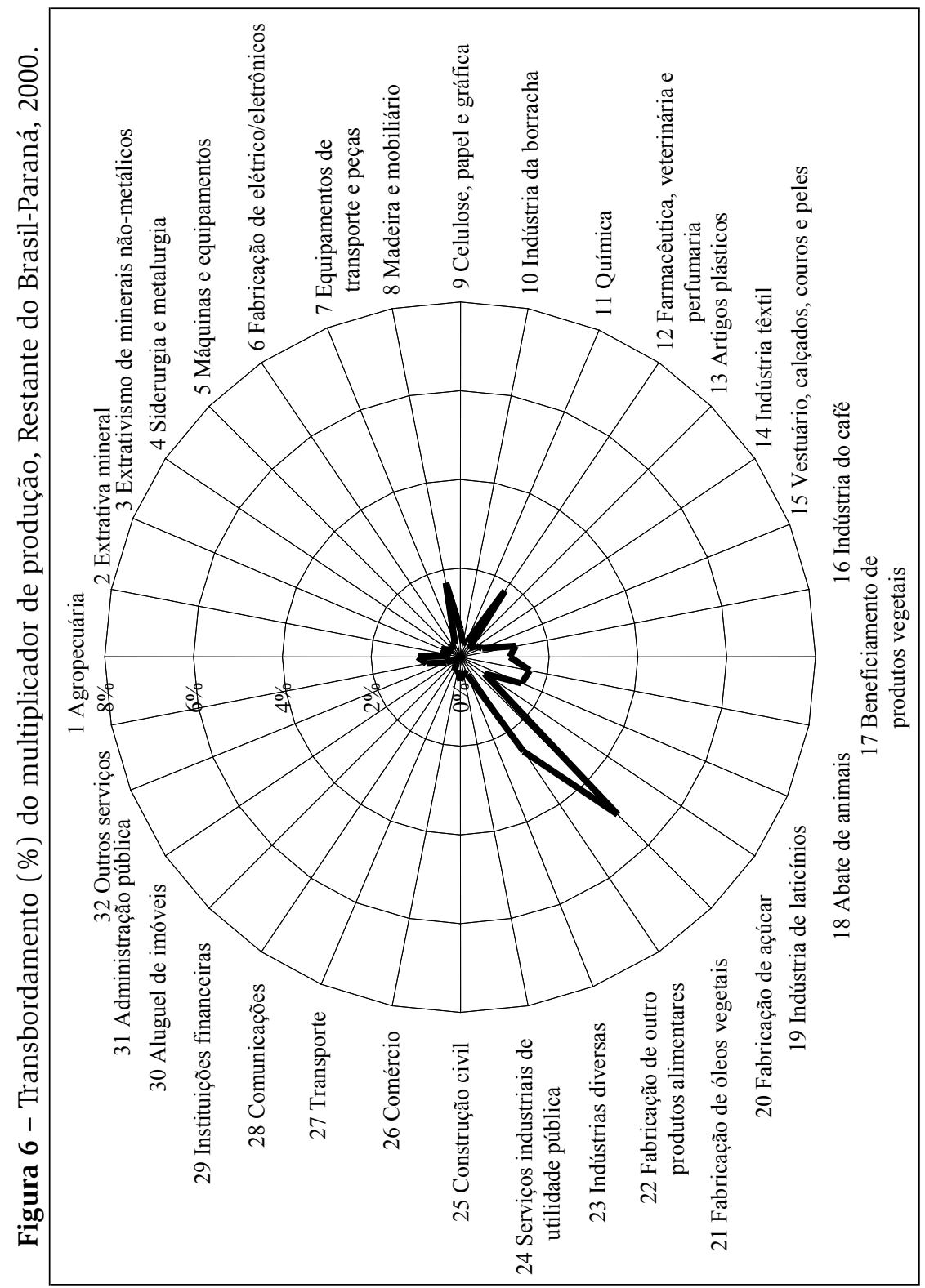

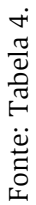




\section{Considerações finais}

Este artigo teve como objetivo estimar e analisar o nível das interações sinérgicas e o transbordamento do efeito multiplicador da produção para 32 setores do sistema inter-regional Paraná-Restante do Brasil nos anos de 1995 e 2000.

As informações são oriundas das matrizes insumo-produto estimadas pelos autores para o Paraná, além da matriz insumo-produção nacional de 1995, construída pelo IBGE (1997), e de 2000, estimada por Guilhoto et al. (2002).

A metodologia utilizada neste artigo ainda é pouco empregada em estudos relativos à economia paranaense e lança luzes aos complexos efeitos sócio-econômicos que as políticas públicas podem gerar.

Os resultados mostraram que a dependência do Paraná em relação ao Restante do Brasil cresceu no período em tela, indicando maior interação comercial entre estas regiões. Em outras palavras, a sinergia entre as regiões se elevou, já que o fluxo de bens e serviços para alimentar o processo produtivo no interior do estado se intensificou no período. Foram encontrados, também, percentuais elevados de transbordamento do efeito multiplicador de produção em vários setores no Paraná, uma vez que a compra de bens intermediários e serviços que o estado fez de outras regiões impactou a produção destas economias.

Não obstante, o elevado efeito transbordamento da produção deve ser visto como um resultado de curto prazo, considerando que a tendência da economia estadual é produzir internamente parcela cada vez maior dos bens e serviços necessários ao seu processo produtivo. No médio e longo prazos, portanto, a exemplo do que ocorreu com a implantação do pólo automobilístico de Betim (MG), a economia paranaense criará as bases para atender às demandas oriundas da expansão, modernização e/ou dos novos setores componentes de sua estrutura produtiva.

Nesse sentido, o poder público, por meio de políticas que visem melhorar a infra-estrutura estadual e de políticas de estímulos setoriais, poderá colaborar, criando o aparato logístico e designando incentivos fiscais e/ou creditícios específicos que auxiliariam a iniciativa privada na implementação de suas atividades e na solução de problemas relativos ao comércio inter-regiões. 
Para estudos futuros, outras variáveis, além da produção, poderiam ser contempladas em trabalhos que objetivem estimar a sinergia e o transbordamento do multiplicador, a exemplo do emprego, da renda e dos impostos. Esses estudos poderiam ser realizados com matrizes estruturadas em maior número de setores, o que permitiria melhor desagregação da estrutura produtiva das regiões e, consequentemente, maior precisão dos indicadores encontrados, fato que superaria uma limitação importante do presente trabalho.

\section{Referências bibliográficas}

ALVES, U. A transformação de R \$8,6 bi. Balanço Anual - Paraná. Gazeta Mercantil, Curitiba, Ano IV, n. 4, p. 610, Agosto 1997.

GUILHOTO, J. J. M.; HEWINGS, G. J. D.; SONIS, M. Synergetic interactions between 2 Brazilian regions: an application of input-output linkages. In: North American Meetings of the RSA, 45, 1998, Santa Fe, New Mexico. Anais... México: RSA, 11-14 nov.

. Productive relations in the Northeast and the rest of Brazil regions in 1992: decomposition \& synergy in input-output systems. In: Encontro Nacional de Economia, 27, 1999, Pará. Anais... Pará: Associação Nacional de Pós-Graduação em Economia, 7 a 10 de dezembro de 1999, p. 1437-1452.

GUILHOTO, J. J. M.; MORETTO, A. C.; RODRIGUES, R. L. Decomposition \& Synergy: a study of the interactions and dependence among the 5 Brazilian macro regions. Economia Aplicada, v. 5, n. 2, p. 345-362, Abril-Junho 2001.

GUILHOTO, J. J. M.; SESSO FILHO, U. A.; LOPES, R. L.; HILGEMBERG, C. M. A. T.; HILGEMBERG, E. M. Nota metodológica: construção da matriz de insumo-produto utilizando dados preliminares das Contas Nacionais. In: Encontro Brasileiro de Estudos Regionais e Urbanos, 2, 2002, São Paulo. Anais...São Paulo, Associação Brasileira de Estudos Regionais e Urbanos, 25 e 26 de outubro de 2002.

INSTITUTO BRASILEIRO DE GEOGRAFIA E ESTATÍSTICA. Matriz de insumo-produto Brasil-1995. Rio de janeiro: IBGE, 1997. 217p. 
IPARDES. PIB do Paraná. 2000. Disponível em < http:/www.pr.gov. br/ipardes/publicacoes/pib/pib2.htm > . Acesso em 13/05/2004.

MILLER, R. E.; BLAIR, P. D. Input-output analysis: foundations and extensions. Englewood Cliffs: Prentice-Hall, 1985. 464p.

MORETTO, A. C. Relações intersetoriais e inter-regionais na economia paranaense em 1995. 2000. 161p. Tese (Doutorado) - Escola Superior de Agronomia Luiz de Queiroz, Universidade de São Paulo, Piracicaba.

MOTIM, B. M.; FIRKOWSKI, O.L.C.F.; ARAÚJO, S.M.P. Desconcentração da indústria brasileira e seus efeitos sobre os trabalhadores: a indústria automobilística no Paraná. Disponível em < http://www. ub.es/geocrit/sn/sn119-88.htm >. Acesso em 04/04/2004.

NUÑES, B. E. C.; KURESKI, R. Contabilidade Social para a economia do Paraná, 2000: Tabelas básicas de insumo-produção. In: Encontro de Economia Paranaense, 2., 2003, Maringá. Anais...Maringá: UEM-UELUEPG-UNIOESTE-IPARDES, 2003, p. 241-259.

OLIVEIRA, M. A. de. Indústria paranaense na década de 1990: reestruturação e concentração. In: Encontro de Economia Paranaense, 2., 2003, Maringá. Anais...Maringá: UEM-UEL-UEPG-UNIOESTE-IPARDES, 2003, p. 499-5169.

PINTO, H. E. M.; MEZA, M. L. F. G. de. Globalização e relações tecnológicas nas empresas multinacionais: o caso Renault do Paraná. In: Encontro de Economia Paranaense, 2., 2003, Maringá. Anais...Maringá: UEM-UEL-UEPG-UNIOESTE-IPARDES, 2003, p. 317-337.

RODRIGUES, R. L. Cooperativas agropecuárias e relações intersetoriais na economia paranaense: uma análise de insumo-produto. 2000. 171p. Tese (Doutorado) - Escola Superior de Agronomia 'Luiz de Queiroz”, Universidade de São Paulo, Piracicaba.

RODRIGUES, R. L.; MORETTO. A. C.; SESSO FILHO, U. A.; SIMÕES, M. C. Sistema inter-regional Paraná-Restante do Brasil: relações sinérgicas e efeitos sobre a produção setorial. . Encontro de Economia Paranaense, 4, 2005, Toledo. Anais...Toledo: UEM-UEL-UEPG-UNIOESTE-IPARDES, 3 e 4 de novembro, 2005. 
SESSO FILHO, U. A.; MORETTO, A. C. ; RODRIGUES, R. L. Interações Sinérgicas e transbordamento do efeito multiplicador de produção das grandes regiões do Brasil. In. Encontro de Economia da Região Sul, 6., 2003, Curitiba. Anais ...Curitiba: Associação Nacional de Pós-Graduação em Economia Sul, 25 e 26 de setembro, 2003, p. 13-32.

SESSO FILHO, U. A.; MORETTO. A. C.; RODRIGUES, R. L.; BALDUCCI, F. L. P.; KURESKI, R. Indústria automobilística no Paraná: impactos na produção local e no Restante do Brasil. Revista Paranaense de Desenvolvimento, n. 106, p. 89-112, jan./jun., 2004.

SIMÕES, M. C.; RODRIGUES, R. L.; MORETTO, A. C.; SESSO FILHO, U. A. Relações comerciais entre o Paraná e o Resto do Brasil nos anos de 1980, 1985, 1990 e 1995. In: Encontro Anual de Iniciação Científica, 12, 2003, Foz do Iguaçu. Anais...Foz do Iguaçu: UNIOESTE, 2 a 5 de setembro, 2003.

SONIS, M.; HEWINGS, G. J. D; MIYAZAWA, K. Synergetic interactions within the pair-wise hierarchy of economic linkages sub-systems. Hitotsubashi Journal of Economics, n. 38, p. 2-17, dez. 1997. 